Research Article

\title{
Study on the Water-Physical Properties of the Cement-Plaster Bonded Rock-Like Materials
}

\author{
Yong Zhang $\mathbb{D}^{1}{ }^{1}$ Zhiguo Cao, ${ }^{1}$ and Xiaomeng Shi ${ }^{2}{ }^{2}$ \\ ${ }^{1}$ State Key Laboratory of Water Resources Protection and Utilization in Coal Mining, Beijing 100011, China \\ ${ }^{2}$ School of Civil Engineering, Beijing Jiaotong University, Beijing 100044, China \\ Correspondence should be addressed to Xiaomeng Shi; shixm@bjtu.edu.cn
}

Received 24 September 2020; Revised 14 January 2021; Accepted 25 January 2021; Published 8 February 2021

Academic Editor: Wen-Chieh Cheng

Copyright (c) 2021 Yong Zhang et al. This is an open access article distributed under the Creative Commons Attribution License, which permits unrestricted use, distribution, and reproduction in any medium, provided the original work is properly cited.

The cement-plaster bonded rock-like material is one of the most commonly used materials to simulate different rocks in physical model tests. However, the applicability of this material in solid-fluid coupling model tests is not clear because there are few research studies on the water-physical properties of this material and its similarity to the actual rock is uncertain. This paper presents a systemic experimental study on the water-physical properties of the cement-plaster bonded rock-like materials. The parameters of rock-like materials, including water absorption, softening coefficient, and permeability coefficient, were compared with those of actual rocks to analyse the applicability of such material. Then, the influence of proportion on the water-physical properties of this material was discussed. By multiple regression analysis of the test results, empirical equations between the waterphysical parameters and proportions were proposed. The equations can be used to estimate the water-physical properties of cement-plaster bonded rock-like materials with specific proportion and thus to select suitable materials in the solid-fluid coupling physical model tests.

\section{Introduction}

The physical model test is a common method to research geotechnical problems in the domains of mining engineering and civil engineering [1-5]. It tries to replicate the geologic body and the structure with an equal or small scale in the laboratory, which could provide a reference for the actual engineering. However, there are still some problems with this approach, one of which is how to make an applicable artificial material to conduct the solid-fluid coupling physical model test [6]. More specifically, it is difficult to select the material with proper water-physical parameters to meet the similarity requirements.

Most of the research studies on solid-fluid coupling materials focus on the nonhydrophilic organic ingredients. Jacoby and Schmeling [7] used glycerol and molten paraffin to simulate mantle convection and plate motion. Kincaid and Olson [8] used paraffin, mineral oil, plaster, and other semiplastic mixed materials as lithosphere to simulate the dynamic process of plate motion. Gong et al. [9] used sand and talcum powder as aggregates and paraffin oil as a cementing agent to simulate the plastically destructive rock mass with low strength and large deformation. Li et al. [10] developed a new type of solid-coupling similar material (PSTO) composed of paraffin, sand, and talcum powder, according to the solid-fluid coupling similarity theory. Huang et al. [11] used quartz sand and bentonite as aggregates and silicone oil and vaseline as bonding materials to develop an artificial material to simulate a waterresisting layer in a solid-fluid coupling physical model test. $\mathrm{Ge}$ and $\mathrm{Xu}$ [12] proposed a method for making a transparent hard rock-like material made of a mixture of rosin saturated solution (RSS), epoxy resin (ER), and curing agent (CA). Although the above materials could meet partial similarity requirements in water-physical properties, the basic mechanical parameters, such as strength and elastic modulus, are significantly different from the actual rocks due to the existence of these organic ingredients. So, this kind of rock-like material is far from an ideal solidfluid coupling material. 
The cement-plaster bonded material is the most commonly used rock-like material because its mechanical behaviors are consistent with actual rocks, and the raw materials are common, cheap, and nontoxic. For this material, the basic properties have been well studied, and there are many application cases [13-15]. However, few studies on the water-physical properties of this material have been reported, and its applicability in the solid-fluid coupling physical model test is uncertain.

In this paper, the water-physical properties of the cement-plaster bonded rock-like material are systematically studied through orthogonal experiments. The applicability of this material is discussed by comparing the water-physical properties of the rock-like material with actual rocks. The multifactors' regression analysis on the test data reveals the relationships between the water-physical properties of the rock-like materials and the proportions of the raw materials. This paper can provide a theoretical basis and reference for the application of such materials in solid-fluid coupling physical model tests.

\section{Similitude Criterion for Water-Physical Parameters}

The water-physical properties of rocks mainly include water content, water absorption, permeability, softening, and frost resistance [16]. Among these parameters, the natural moisture content can be controlled through the curing conditions, and the frost resistance was rarely involved in the physical modelling. Therefore, this study only focuses on three water-physical parameters: water absorption $w_{a}$, permeability coefficient $k$, and softening coefficient $\eta_{c}$.

For most physical modelling tests, the model is always reduced to scale with the protype. The ratios of the parameters between the protype and the model are defined as the similitude scales.

Water absorption $w_{a}$ is the proportion of water which can be absorbed by rock under specific immersion conditions. It can be calculated as follows:

$$
w_{a}=\frac{m_{o}-m_{\mathrm{dr}}}{m_{\mathrm{dr}}} \times 100 \%,
$$

where $m_{o}$ is the saturated quality and $m_{\mathrm{dr}}$ is the dried quality.

The water absorption $w_{a}$ is dimensionless, so the similitude scale is $C_{w}=1$, which means the water absorption $w_{a}$ of the rock-like material should be equal to that of actual rock.

Permeability is the property of rocks that is an indication of the ability for fluids to flow through rocks. According to the similarity theory [1], the similitude scale of permeability coefficient $k$ is as follows:

$$
C_{k}=\frac{\sqrt{C_{l}}}{C_{\gamma}},
$$

where $C_{l}$ and $C_{\gamma}$ are similitude scales of geometry and weight, which are given parameters in the tests.
The softening coefficient $\eta_{c}$ is the ratio of the compressive strength of the rock in the saturated state $\sigma_{\mathrm{cw}}$ to that in the dried state $\sigma_{c}$ :

$$
\eta_{c}=\frac{\sigma_{\mathrm{cw}}}{\sigma_{c}}
$$

Since the softening coefficient is dimensionless, the similitude scale is $C_{\eta}=1$.

In the following experiments, these similitude scales are an important basis for discussing the similarity of such materials to the actual rocks.

\section{Orthogonal Experiment on the Water- Physical Properties of Rock-Like Materials}

3.1. Raw Component Materials. The rock-like materials are composed of cement, plaster, and quartz sand and mixed by water. The cement and plaster are the bonding materials, and the quartz sand is the aggregate. Table 1 lists the characteristics of the raw materials.

3.2. Experiment Design and Process. The orthogonal experimental design method has been widely used in the experimental design, especially in multifactor experiments. This method has obvious advantages compared with other methods in proportioning tests and is commonly used in the research on the ratio of rock-like artificial materials $[17,18]$.

In this experiment, $A / B$ (the ratio of the aggregate to bonding materials), $C / B$ (the percentage of cement in bonding materials), and GS (the grain size of the quartz sand) are defined as the three critical factors in this orthogonal experiment, and each factor has four levels. The orthogonal experimental scheme is shown in Table 2.

According to the orthogonal experimental design method [17], the detailed proportions of rock-like materials for all the test conditions are shown in Table 3.

Making the specimens of the rock-like material is the first step of this experiment. Figure 1 shows the mould of the specimen with the inside dimension $\Phi 50 \times 100 \mathrm{~mm}$. There are seven specimens for each proportion, numbered $i-1 \sim i-7$, where $i$ is the test number, as shown in Table 3 . Figure 2 shows the specimens of cement-plaster bonded rock-like materials.

The uniaxial compressive test was carried out on no. $i-1$ to $i-3$ specimens to obtain the compressive strength in naturally air-dried state $\sigma_{c}$. The specimens numbered $i-4$ to $i-6$ were dried and then weighed to get the drying quality $m_{\mathrm{dr}}$. Then, the permeability coefficient $k$ of the specimens $i-4$ to $i-6$ was measured by penetration tests. The saturated specimens were weighed to obtain saturated quality $m_{o}$. At last, the uniaxial compressive tests were carried out again on the saturated specimens $i-4$ to $i-6$ to obtain the compressive strength in the saturated state $\sigma_{\mathrm{cw}}$. According to equations (1) and (3), the water absorption $w_{a}$ and softening coefficient $\eta_{c}$ of the specimen are calculated. The test results are shown in Table 4. 
TABLE 1: Characteristics of raw component materials.

\begin{tabular}{lc}
\hline Raw materials & Components and types \\
\hline $\begin{array}{l}\text { Quartz sand } \\
\text { Cement }\end{array}$ & $\mathrm{SiO}_{2}>95 \%$ \\
Plaster & Ordinary Portland cement \\
\hline
\end{tabular}

TABLE 2: Factors and levels in the orthogonal experiment.

\begin{tabular}{lccc}
\hline Level & $A / B$ & $C / B(\%)$ & $G S(\mathrm{~mm})$ \\
\hline 1 & $2: 1$ & 0 & $0.25 \sim 0.50$ \\
2 & $4: 1$ & 33 & $0.5 \sim 1$ \\
3 & $6: 1$ & 66 & $1 \sim 2$ \\
4 & $8: 1$ & 100 & $2 \sim 4$ \\
\hline
\end{tabular}

TABLE 3: Orthogonal experiment schemes.

\begin{tabular}{lccc}
\hline Number $i$ & $A / B$ & $C / B(\%)$ & $G S(\mathrm{~mm})$ \\
\hline 1 & $2: 1$ & 0 & $0.25 \sim 0.50$ \\
2 & $2: 1$ & 33 & $0.5 \sim 1$ \\
3 & $2: 1$ & 66 & $1 \sim 2$ \\
4 & $2: 1$ & 100 & $2 \sim 4$ \\
5 & $4: 1$ & 0 & $0.5 \sim 1$ \\
6 & $4: 1$ & 33 & $0.25 \sim 0.50$ \\
7 & $4: 1$ & 66 & $2 \sim 4$ \\
8 & $4: 1$ & 100 & $1 \sim 2$ \\
9 & $6: 1$ & 0 & $1 \sim 2$ \\
10 & $6: 1$ & 33 & $2 \sim 4$ \\
11 & $6: 1$ & 66 & $0.25 \sim 0.50$ \\
12 & $6: 1$ & 100 & $0.5 \sim 1$ \\
13 & $8: 1$ & 0 & $2 \sim 4$ \\
14 & $8: 1$ & 33 & $1 \sim 2$ \\
15 & $8: 1$ & 66 & $0.5 \sim 1$ \\
16 & $8: 1$ & 100 & $0.25 \sim 0.50$ \\
\hline
\end{tabular}

\section{Similarity Analysis between the Rock-Like Materials and Actual Rocks}

Figure 3 shows the failure pattern of the cement-plaster bonded rock-like material in the saturated state, which is similar to that of the actual rocks. By comparing the waterphysical properties of rock-like materials, as shown in $\mathrm{Ta}$ ble 4 , with the properties of actual rocks, as shown in Table 5 [16], the similarity could be analysed. The variation range of water absorption $w_{a}$ is $13.5 \% \sim 30.3 \%$, softening coefficient $\eta_{c}$ is $0.421 \sim 0.713$, and permeability coefficient $k$ is $1.62 \times 10^{-6} \sim 4.66 \times 10^{-4} \mathrm{~cm} / \mathrm{s}$.

The water absorptions of actual rocks do not exceed $10 \%$. Obviously, the rock-like materials absorbed much more water than the actual rocks. So, this kind of material cannot meet the requirement of similarity of water absorption. In other words, the cement-plaster bonded rock-like material could not be used in a physical modelling, if the water absorption was defined as a key influencing factor or an important research subject.

The softening coefficient of rock-like materials is similar with that of the actual rocks, especially for the medium-hard rock and soft rock. But, for the hard rocks with a softening coefficient more than 0.7 , the rock-like material could not simulate well.

The permeability coefficient of the rock varies over a wide range. By comparing the data, the rock-like material can be used to simulate limestone and sandstone with more cracks and pores. For the dense rock, the permeability coefficient is too small to be simulated by such material.

In general, the softening and permeability coefficients of this material are relatively similar to those of actual rocks, and this material is suitable for the simulation of soft or medium-hard rocks with cracks or pores. However, in the physical modelling tests where water absorption is a key influencing factor or an important research subject, the use of this material is not recommended because of the difference in water absorption.

\section{Influence of Proportions on the Water- Physical Properties}

5.1. Water Absorption. According to the orthogonal experiment theory, the mean values and the variation ranges of the water absorption for each factor at each level are calculated, and the results are shown in Table 6 . The intuitive analysis diagram is shown in Figure 4. It can be seen that the change of each factor could lead to the variation of water absorption. GS is the most sensitive factor. The water absorption increases with the increase of the $A / B$, decreases with the rise of $C / B$, and increases significantly with the increase of GS.

5.2. Softening Coefficient. The mean values and the variation ranges of the softening coefficient for each factor at each level are calculated, and the results are shown in Table 7. The intuitive analysis diagram is shown in Figure 5. Apparently, the softening coefficient increases significantly with the rise of $C / B$, while $A / B$ and $G S$ have little effect on it. The reason is that the strength of the gypsum in the bonding material is very much affected by water. This leads to a smaller softening coefficient for materials with a higher gypsum content.

5.3. Permeability Coefficient. The mean values and the variation ranges of the permeability coefficient for each factor at each level are calculated, and the results are shown in Table 8. The intuitive analysis diagram is shown in Figure 6. The effect of GS on the permeability coefficient is the most obvious, and the permeability coefficient increases significantly with the increase of $G S$. The $A / B$ and $C / B$ also have some influence on the permeability coefficient. It increases with the rise of $A / B$ and decreases with the increase of $C / B$.

\section{Multifactors Regression Analysis}

The above intuitive analysis diagrams show that, except for the exponential relationship between the permeability coefficient and the GS, all other factors and the water-physical parameters can be described by the linear relationship. Define $A / B$ as $X_{1}, C / B$ as $X_{2}$, and the mean values of $G S$ as $X_{3}$. 


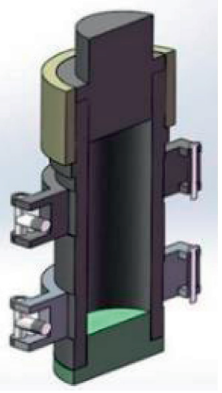

(a)

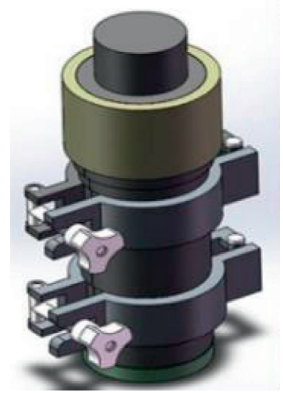

(b)

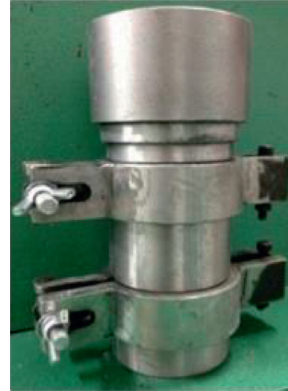

(c)

Figure 1: The specimen mould.

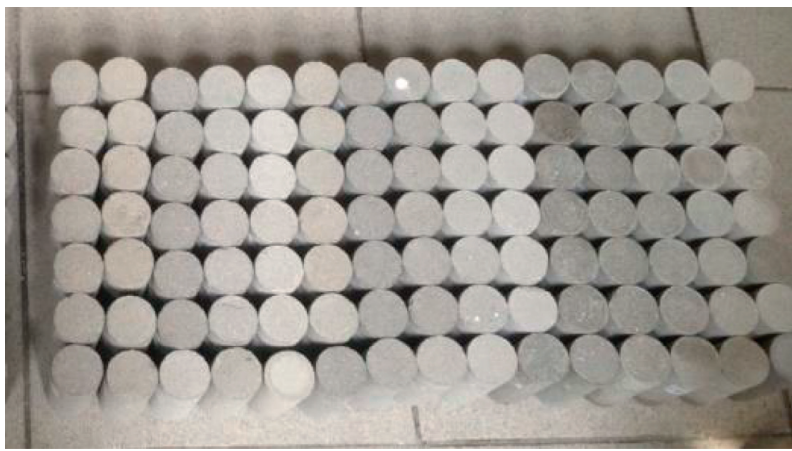

FIGURE 2: The maintained specimen.

TABle 4: Orthogonal experiment results of the similar material ratio.

\begin{tabular}{|c|c|c|c|c|c|c|c|c|c|c|c|c|}
\hline \multirow{2}{*}{ Number } & \multicolumn{4}{|c|}{ Water absorption $w_{a}(\%)$} & \multicolumn{4}{|c|}{ Softening coefficient $\eta_{c}$} & \multicolumn{4}{|c|}{ Permeability coefficient $k\left(10^{-5} \mathrm{~cm} / \mathrm{s}\right)$} \\
\hline & I & II & III & Mean & I & II & III & Mean & I & II & III & Mean \\
\hline 1 & 15.1 & 16.2 & 16.1 & 15.8 & 0.477 & 0.482 & 0.469 & 0.476 & 0.098 & 0.256 & 0.132 & 0.162 \\
\hline 2 & 17.6 & 17.8 & 18.6 & 18.0 & 0.505 & 0.498 & 0.503 & 0.502 & 0.355 & 0.398 & 0.453 & 0.402 \\
\hline 3 & 18.8 & 19.6 & 19.2 & 19.2 & 0.633 & 0.641 & 0.64 & 0.638 & 4.068 & 4.003 & 3.824 & 3.965 \\
\hline 4 & 21.1 & 20.8 & 19.6 & 20.5 & 0.708 & 0.701 & 0.703 & 0.704 & 10.085 & 8.987 & 8.624 & 9.232 \\
\hline 5 & 21.5 & 20.2 & 21.3 & 21.0 & 0.506 & 0.503 & 0.494 & 0.501 & 0.878 & 0.968 & 0.926 & 0.924 \\
\hline 6 & 13.2 & 14.5 & 13.1 & 13.6 & 0.589 & 0.581 & 0.579 & 0.583 & 0.312 & 0.485 & 0.391 & 0.396 \\
\hline 7 & 22.2 & 21.8 & 22.9 & 22.3 & 0.601 & 0.605 & 0.600 & 0.602 & 28.069 & 29.622 & 31.268 & 29.653 \\
\hline 8 & 19.8 & 20.8 & 19.7 & 20.1 & 0.622 & 0.618 & 0.629 & 0.623 & 8.658 & 7.652 & 7.285 & 7.865 \\
\hline 9 & 26.1 & 27.1 & 26.3 & 26.5 & 0.441 & 0.451 & 0.446 & 0.446 & 9.712 & 10.989 & 9.134 & 9.945 \\
\hline 10 & 30.5 & 30.1 & 30.3 & 30.3 & 0.481 & 0.485 & 0.498 & 0.488 & 38.956 & 39.861 & 41.339 & 40.052 \\
\hline 11 & 13.0 & 14.1 & 13.4 & 13.5 & 0.628 & 0.633 & 0.635 & 0.632 & 0.751 & 0.621 & 0.584 & 0.652 \\
\hline 12 & 16.8 & 16.9 & 17.9 & 17.2 & 0.712 & 0.725 & 0.702 & 0.713 & 1.389 & 1.847 & 1.687 & 1.641 \\
\hline 13 & 30.0 & 31.0 & 29.9 & 30.3 & 0.415 & 0.42 & 0.428 & 0.421 & 49.698 & 41.295 & 48.846 & 46.613 \\
\hline 14 & 26.5 & 26.8 & 27.7 & 27.0 & 0.556 & 0.543 & 0.551 & 0.550 & 13.369 & 12.087 & 12.803 & 12.753 \\
\hline 15 & 20.1 & 21.1 & 20.9 & 20.7 & 0.561 & 0.568 & 0.557 & 0.562 & 2.611 & 2.148 & 2.945 & 2.568 \\
\hline 16 & 16.6 & 16.5 & 15.8 & 16.3 & 0.687 & 0.692 & 0.691 & 0.690 & 0.772 & 0.821 & 0.948 & 0.847 \\
\hline
\end{tabular}

Define water absorption $w_{a}$ as $Y_{1}$, softening coefficient $\eta_{c}$ as $Y_{2}$, and permeability coefficient $k$ as $Y_{3}$. Multifactors' regression analysis was carried out on the orthogonal test data of all 16 groups of rock-like materials in Table 3 , and the results are shown as follows:

$$
\left.\begin{array}{l}
Y_{1}=0.126+0.010 X_{1}-0.045 X_{2}+0.039 X_{3} \\
Y_{2}=0.498-0.004 X_{1}+0.220 X_{2}-0.011 X_{3} \\
Y_{3}=-16.762+2.115 X_{1}-7.898 X_{2}+7.874 e^{0.548 X_{3}}
\end{array}\right\} \text {. }
$$




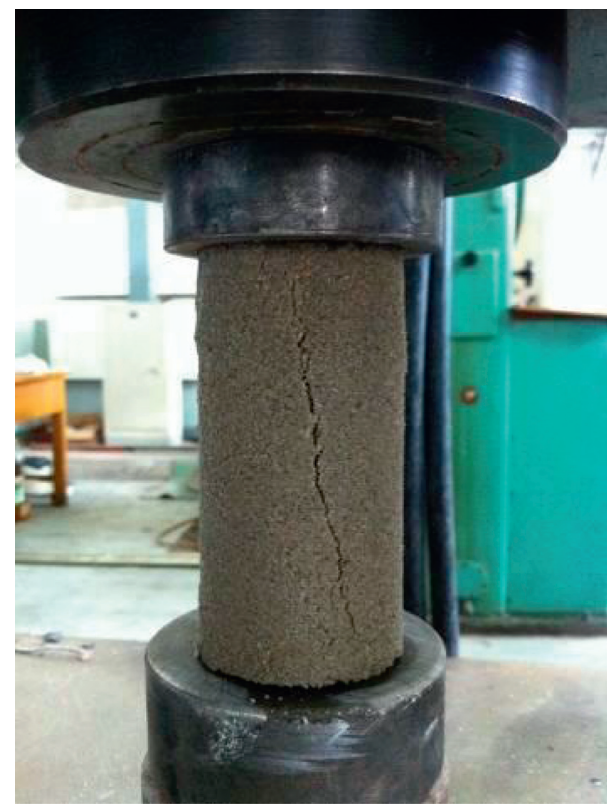

FIgURE 3: Failure of a saturated specimen.

TABLe 5: Water-physical properties of common rocks.

\begin{tabular}{lcccc}
\hline Rock type & Water absorption $w_{a}(\%)$ & Softening coefficient $\eta_{c}$ & Cracks and pores & Permeability coefficient $(\mathrm{cm} / \mathrm{s})$ \\
Granite & $0.1 \sim 0.4$ & $0.80 \sim 0.98$ & $\begin{array}{c}\text { Dense and microcracks } \\
\text { Microcracks } \\
\text { Coarse cracks }\end{array}$ & $\begin{array}{c}1.1 \times 10^{-12} \sim 9.5 \times 10^{-11} \\
1.1 \times 10^{-11} \sim 2.5 \times 10^{-11} \\
2.8 \times 10^{-9} \sim 7 \times 10^{-8}\end{array}$ \\
\hline Limestone & $0.1 \sim 4.5$ & Dense & $\begin{array}{c}3 \times 10^{-12} \sim 6 \times 10^{-10} \\
2 \times 10^{-9} \sim 3 \times 10^{-6} \\
9 \times 10^{-5} \sim 3 \times 10^{-4}\end{array}$ \\
\hline Sandstone & $0.2 \sim 9.0$ & $0.68 \sim 0.94$ & Microcracks and micropore & Porous \\
\hline Shale & $0.5 \sim 3.2$ & $0.60 \sim 0.97$ & Dense & $10^{-13} \sim 2.5 \times 10^{-12}$ \\
\hline
\end{tabular}

TABLE 6: Extremum difference analysis of water absorption.

\begin{tabular}{|c|c|c|c|c|c|}
\hline \multirow{2}{*}{ Factor } & \multicolumn{5}{|c|}{ Water absorption $w_{a}(\%)$} \\
\hline & Level 1 & Level 2 & Level 3 & Level 4 & Range \\
\hline$A / B$ & 18.4 & 19.3 & 21.9 & 23.6 & 5.2 \\
\hline$C / B$ & 23.4 & 22.2 & 18.9 & 18.5 & 4.9 \\
\hline$G S$ & 14.8 & 19.2 & 23.2 & 25.9 & 11.1 \\
\hline
\end{tabular}

For these three regression equations, the correlation coefficients are $0.812,0.855$, and 0.832 , respectively. The relationship between the proportion of materials and the water-physical properties can be described quantitatively by the above regression equations, which can be used to estimate the water-physical properties of cement-plaster 


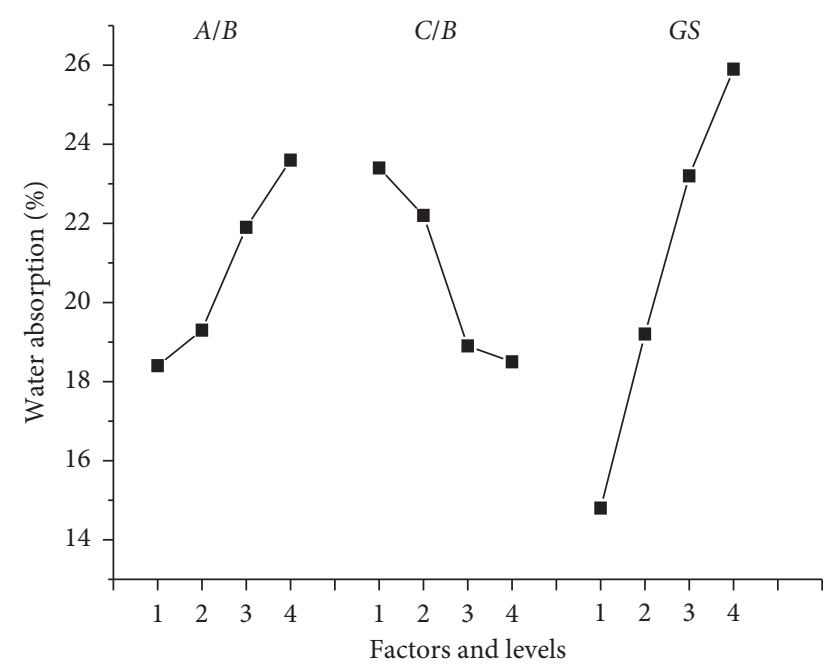

FIgURE 4: Sensitivity analysis of water absorption.

TABLE 7: Extremum difference analysis of the softening coefficient.

\begin{tabular}{lccccc}
\hline \multirow{2}{*}{ Factor } & \multicolumn{5}{c}{ Softening coefficient $\eta_{c}$} \\
& Level 1 & Level 2 & Level 3 & Level 4 & Range \\
\hline$A / B$ & 0.580 & 0.577 & 0.570 & 0.556 & 0.024 \\
$C / B$ & 0.461 & 0.531 & 0.609 & 0.682 & 0.221 \\
$G S$ & 0.595 & 0.570 & 0.564 & 0.554 & 0.041 \\
\hline
\end{tabular}

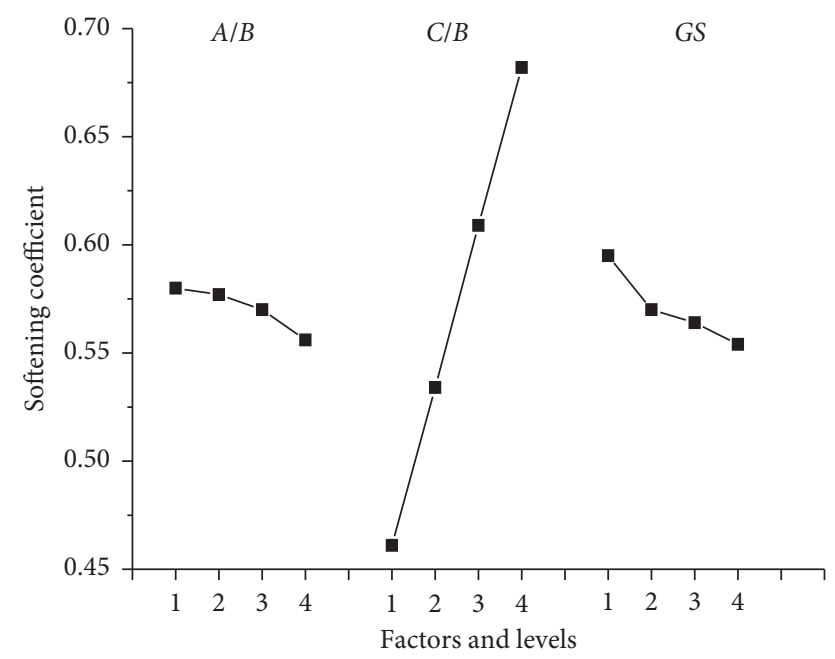

FIgURE 5: Sensitivity analysis of the softening coefficient.

TABLE 8: Extremum difference analysis of the permeability coefficient.

\begin{tabular}{lccccc}
\hline \multirow{2}{*}{ Factor } & \multicolumn{5}{c}{ Permeability coefficient $k\left(10^{-5} \mathrm{~cm} / \mathrm{s}\right)$} \\
& Level 1 & Level 2 & Level 3 & Level 4 & Range \\
\hline$A / B$ & 3.440 & 9.710 & 13.072 & 15.695 & 12.255 \\
$C / B$ & 14.411 & 13.401 & 9.209 & 4.896 & 9.515 \\
$G S$ & 0.514 & 1.384 & 8.632 & 31.387 & 30.873 \\
\hline
\end{tabular}

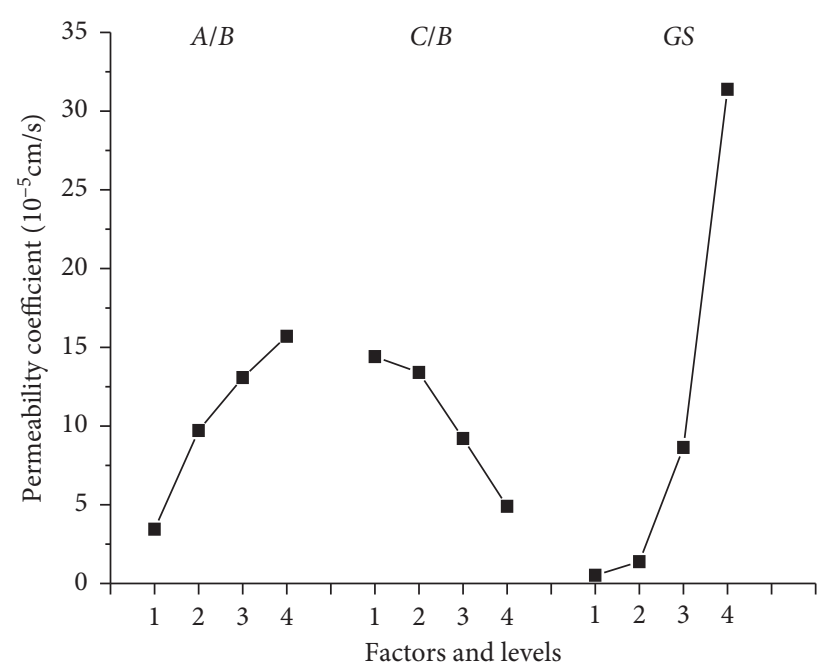

FIGURE 6: Sensitivity analysis of the permeability coefficient.

bonded rock-like materials with specific proportion and thus to select suitable materials in the solid-fluid coupling physical model tests.

\section{Conclusions}

(1) The failure pattern of the cement-plaster bonded rock-like material in the saturated state is similar to that of the actual rocks. For this material, the variation range of water absorption $w_{a}$ is $13.5 \% \sim 30.3 \%$, softening coefficient $\eta_{c}$ is $0.421 \sim 0.713$, and permeability coefficient $k$ is $1.62 \times 10^{-6} \sim 4.66 \times 10^{-4} \mathrm{~cm} / \mathrm{s}$.

(2) The softening and permeability coefficients of this material are relatively similar to those of actual rocks, and this material is suitable for the simulation of soft or medium-hard rocks with cracks or pores. However, in the physical modelling tests where water absorption is a key influencing factor or an important research subject, the use of this material is not recommended because of the difference in water absorption.

(3) The water absorption increases with the increase of the $A / B$, decreases with the rise of $C / B$, and increases significantly with the increase of $G S$. The softening coefficient increases significantly with the rise of $C / B$, while $A / B$ and $G S$ have little effect on it. The permeability coefficient increases significantly with the increase of $G S$, increases with the rise of $\mathrm{A} / \mathrm{B}$, and decreases with the increase of $\mathrm{C} / \mathrm{B}$.

(4) The relationship between the proportion of materials and the water-physical properties can be described quantitatively by the regression equations based on the orthogonal test results. The equations can be used to estimate the water-physical properties of cementplaster bonded rock-like materials with specific proportion and thus to select suitable materials in the solid-fluid coupling physical model tests. 


\section{Data Availability}

The data used to support the findings of this study are available from the corresponding author upon request.

\section{Conflicts of Interest}

The authors declare that they have no conflicts of interest regarding the publication of this paper.

\section{Acknowledgments}

This work was supported by the Open Fund of State Key Laboratory of Water Resource Protection and Utilization in Coal Mining (Grant no. GJNY-18-73.2) and Science and Technology Innovation Project of China Energy Investment Corporation (SHJT-16-26).

\section{References}

[1] D. Z. Gu, Similar Materials and Similar Models, China University of Mining and Technology Press, Xuzhou, China, 1995.

[2] Y. M. Lin, Experimental Rock Mechanics - Physical Modelling, China Coal Industry Publishing House, Beijing, China, 1984.

[3] M. C. He, W. L. Gong, H. M. Zhai, and H. P. Zhang, "Physical modeling of deep ground excavation in geologically horizontal strata based on infrared thermography," Tunnelling and Underground Space Technology, vol. 25, no. 4, pp. 366-376, 2010.

[4] J. Liu, X.-T. Feng, X.-L. Ding, J. Zhang, and D.-M. Yue, "Stability assessment of the Three-Gorges Dam foundation, China, using physical and numerical modeling-Part I: physical model tests," International Journal of Rock Mechanics and Mining Sciences, vol. 40, no. 5, pp. 609-631, 2003.

[5] Z. K. Li, Q. J. Xu, G. F. Luo et al., "3D geo-mechanical model test for large scaled underground hydropower station," Journal of Hydraulic Engineering, vol. 33, no. 5, pp. 31-36, 2002.

[6] J. Zhang and Z. J. Hou, "Experimental study on simulation materials for solid-liquid coupling," Chinese Journal of Rock Mechanics and Engineering, vol. 23, no. 18, pp. 3157-3161, 2004.

[7] W. R. Jacoby and H. Schmeling, "Convection experiments and the driving mechanism," Geologische Rundschau, vol. 70, no. 1 , pp. 207-230, 1981.

[8] C. Kincaid and P. Olson, "An experimental study of subduction and slab migration," Journal of Geophysical Research: Solid Earth, vol. 92, no. B13, pp. 13832-13840, 1987.

[9] Z. X. Gong, C. M. Guo, and D. S. Gao, "The experiment studies of the geomechanical model material," Journal of Yangtze River Scientific Research Institute, vol. 10, no. 1, pp. 32-46, 1984.

[10] S. C. Li, X. D. Feng, S. C. Li et al., "Research and development of a new similar material for solid-fluid coupling and its application," Chinese Journal of Rock Mechanics and Engineering, vol. 29, no. 2, pp. 281-288, 2010.

[11] Q. X. Huang, W. Z. Zhang, and Z. C. Hou, "Study of simulation materials of aquifuge for solid-liquid coupling," Chinese of Journal of Rock Mechanics and Engineering, vol. 29, no. S1, pp. 2813-2818, 2010.

[12] J. Ge and Y. Xu, "A method for making transparent hard rocklike material and its application," Advances in Materials
Science and Engineering, vol. 2019, pp. 1-14, Article ID 1274171, 2019.

[13] X. M. Shi, B. G. Liu, Y. Y. Xiang, and Y. Qi, "A method for selecting similar materials for rocks in scaled physical modeling tests," Journal of Mining Science, vol. 54, no. 6, pp. 938-948, 2018.

[14] X. M. Shi, B. G. Liu, and J. Xiao, "A method for determining the ratio of similar materials with cement and plaster as bonding agents," Rock and Soil Mechanics, vol. 36, no. 5, pp. 1357-1362, 2015.

[15] P. P. Luo, S. R. Wang, P. Hagan et al., "Mechanical performances of cement-gypsum composite material containing a weak interlayer with different angles," DYNA, vol. 94, no. 4, pp. 447-454, 2019.

[16] M. F. Cai, Rock Mechanics and Rock Engineering, Science Press, Beijing, China, 2002.

[17] J. Y. Dong, J. H. Yang, G. X. Yang et al., "Research on similar material proportioning test of model test based on orthogonal design," Journal of China Coal Society, vol. 37, no. 1, pp. 44-79, 2012.

[18] H. C. Zhu and M. W. Xie, "The way using orthogonal experiment to make up a prescription of similar materials," Journal of Wuhan University of Hydraulic and Electric Engineering, vol. 23, no. 4, pp. 103-109, 1990. 